\title{
Supernova constraints on dark flavored sectors
}

\author{
Jorge Martin Camalich®, ${ }^{1,2}$ Jorge Terol-Calvo ${ }^{1,2,3}$ Laura Tolos $\odot,{ }^{4,5,6,7}$ and Robert Ziegler ${ }^{8}$ \\ ${ }^{1}$ Instituto de Astrofísica de Canarias, C/ Vía Láctea, s/n E38205-La Laguna (Tenerife), Spain \\ ${ }^{2}$ Universidad de La Laguna, Departamento de Astrofísica, La Laguna, E-38206 Tenerife, Spain \\ ${ }^{3}$ Instituto de Física Corpuscular (CSIC-Universitat de València), \\ C/ Catedrático José Beltrán 2, E-46980 Paterna (València), Spain \\ ${ }^{4}$ Institute of Space Sciences (ICE, CSIC), Campus UAB, Carrer de Can Magrans, 08193 Barcelona, Spain \\ ${ }^{5}$ Institut d'Estudis Espacials de Catalunya (IEEC), 08034 Barcelona, Spain \\ ${ }^{6}$ Frankfurt Institute for Advanced Studies, Ruth-Moufang-Str. 1, 60438 Frankfurt am Main, Germany \\ ${ }^{7}$ Faculty of Science and Technology, University of Stavanger, 4036 Stavanger, Norway \\ ${ }^{8}$ Institut für Theoretische Teilchenphysik, Karlsruhe Institute of Technology, 76131 Karlsruhe, Germany
}

(Received 11 January 2021; revised 24 March 2021; accepted 3 May 2021; published 4 June 2021)

\begin{abstract}
Proto-neutron stars forming a few seconds after core-collapse supernovae are hot and dense environments where hyperons can be efficiently produced by weak processes. By making use of various state-of-the-art supernova simulations combined with the proper extensions of the equations of state including $\Lambda$ hyperons, we calculate the cooling of the star induced by the emission of dark particles $X^{0}$ through the decay $\Lambda \rightarrow n X^{0}$. Comparing this novel energy-loss process to the neutrino cooling of SN 1987A allows us to set a stringent upper limit on the branching fraction, $\operatorname{BR}\left(\Lambda \rightarrow n X^{0}\right) \leq 8 \times 10^{-9}$, that we apply to massless dark photons and axions with flavor-violating couplings to quarks. We find that the new supernova bound can be orders of magnitude stronger than other limits in dark-sector models.
\end{abstract}

DOI: 10.1103/PhysRevD.103.L121301

\section{INTRODUCTION}

Clarifying the fundamental nature of dark matter remains one of the major challenges of modern physics [1]. An attractive possibility is to postulate the existence of a dark sector, neutral under the Standard Model (SM) gauge group and interacting with ordinary matter through new mediators or portals. Dark photons (or light $Z^{\prime}$-bosons) induced by hidden gauge groups [2,3], or axions and axionlike-particles (ALPs) arising from a spontaneously broken global symmetry [4-11] are prime examples of bosonic portals (see [12] for a review). Indeed, dark-sector scenarios have attracted much attention over the past years, leading to an extensive experimental program to search for feebly interacting particles [13-16].

If the dark photon is strictly massless, then it can interact with the SM fields only through higher-dimension operators whose structure ultimately depend on the ultraviolet (UV) completion of the model [17]. It can couple to fermions of all generations and, in general, mediates flavor-changing processes [17-23] (see [3] for a review). Also axions and ALPs can display a rich flavor structure

Published by the American Physical Society under the terms of the Creative Commons Attribution 4.0 International license. Further distribution of this work must maintain attribution to the author(s) and the published article's title, journal citation, and DOI. Funded by SCOAP . depending, again, on the UV dynamics of the model and, in particular, in scenarios addressing also the flavor puzzle [24-30]. Hence, rare meson and lepton decays or meson-mixing can pose serious constraints in these models $[3,26,29,30]$. On the other hand, energy-loss arguments applied to stellar evolution lead to some of the strongest indirect bounds on dark sectors [31-35]. They typically constrain the emission of particles that couple to photons, electrons or nucleons, and with masses below the temperature of the stellar plasma [35,36]. A particularly interesting system is the proto-neutron star (PNS) forming during corecollapse supernovae (SN) [37], which reaches temperatures and densities that enable the production of muons [38] or $\Lambda$ hyperons [39]. This opens up the possibility to probe the couplings of the dark sector to heavier flavors of the SM $[29,40,41]$.

The observation of SN 1987A (and possibly of NS 1987A $[42,43]$ ) has helped to confirm the standard picture of core-collapse SN [44-46] (see however Ref. [47] for a critical view). An experimental limit on dark luminosity stems from the observation of a neutrino pulse, sustained over $\sim 10 \mathrm{~s}[48,49]$, in coincidence with $\mathrm{SN}$ 1987A [50-52]. Exotic cooling would shorten the neutrino signal, leading to the classical bound [35],

$$
L_{\mathrm{d}} \lesssim 3 \times 10^{52} \mathrm{erg} \mathrm{s}^{-1},
$$

at $\sim 1 \mathrm{~s}$ after bounce (see also [53-63]). 
In the present letter we discuss the possibility that also hyperons can contribute to the dark luminosity through the decay process $\Lambda \rightarrow n X^{0}$ if the dark particles, $X^{0}$, interact with strange quarks. This idea was first explored in [29] for the case that $X^{0}$ is a flavor-violating QCD axion. Here we investigate this novel SN cooling mechanism by implementing state-of-the-art simulations combined with proper extensions of the nuclear equations of state (EoS) to include $\Lambda$ 's. This allows us to set an upper limit on the branching fraction of the decay that, for definiteness (and simplicity), is applied to the case in which $X^{0}$ is a massless dark photon or an axion. As we will discuss below, the new SN bound on these models can be orders of magnitude stronger than those obtained from other sources.

\section{EMISSION RATES}

The width of the decay $\Lambda \rightarrow n X^{0}$ for a massless $X^{0}$, in vacuum and the $\Lambda$ 's rest frame reads

$$
\Gamma \equiv \Gamma\left(\Lambda \rightarrow n X^{0}\right)=\frac{\bar{\omega}^{3}}{2 \pi} C_{X},
$$

where $\bar{\omega}=\left(m_{\Lambda}^{2}-m_{n}^{2}\right) / 2 m_{\Lambda}$ is the $X^{0}$ energy in this frame, $m_{\mathfrak{B}}(\mathfrak{B}=n, \Lambda)$ are the baryon masses and $C_{X}$ is a constant with dimensions of $E^{-2}$ that is related to the energy scale and couplings of the model. The spectrum of the emission rate per unit volume that is induced by this process in the medium is given by

$$
\frac{d \mathcal{N}_{\mathrm{em}}}{d \omega}=\frac{m_{\Lambda}^{2} \Gamma}{2 \pi^{2} \bar{\omega}} \int_{E_{0}}^{\infty} d E f_{\Lambda}\left(1-f_{n}\right),
$$

where $\omega(E)$ is the energy of the $X^{0}(\Lambda)$ in the PNS's rest frame. The number densities of the baryons follow the relativistic Fermi distributions, $f_{\mathfrak{B}}$, at a given temperature, $T$, and chemical potential, $\mu$, established by " $\beta$-equilibrium", $p e^{-} \leftrightarrow \mathfrak{B} \nu_{e}$. In Eq. (3) we have neglected a Bose-stimulation factor $\left(1+f_{X}\right)$ where $f_{X}$ is now a BoseEinstein distribution. Finally, $E_{0}=m_{\Lambda}\left(\omega^{2}+\bar{\omega}^{2}\right) /(2 \omega \bar{\omega})$ is the minimal energy of the $\Lambda$ required to produce an $X^{0}$ with energy $\omega$. By multiplying Eq. (3) by $\omega$ one derives the spectrum of the energy-loss rate $d Q / d \omega$, which integrated over $\omega$ gives the total rate of energy radiated by the star per unit volume of the stellar plasma.

An approximate (and more intuitive) formula can be obtained by neglecting the Pauli-blocking for neutrons and taking the limit where $E, \omega, m_{\Lambda}-m_{n}$ are all much smaller than $m_{n}$ in Eq. (3) [29],

$$
Q \simeq n_{\Lambda}\left(m_{\Lambda}-m_{n}\right) \Gamma,
$$

where $n_{\Lambda}$ is the number density of $\Lambda$ in the medium. If we further neglect interactions of the baryons with the medium, so that $\Lambda$ 's are only produced via thermal fluctuations at given chemical potential, then

$$
n_{\Lambda} \simeq n_{n} \exp \left(-\frac{m_{\Lambda}-m_{n}}{T}\right) .
$$

There are other mechanisms that produce $X^{0}$ from the $\Lambda n$ coupling, such as the bremsstrahlung process $\Lambda n \rightarrow n n X^{0}$. As we will see below, production by $\Lambda \rightarrow n X^{0}$ decays always leads to stronger bounds on the $X^{0}$ couplings than the corresponding process in nucleons, like $n n \rightarrow n n X^{0}$. Since replacing an initial neutron by a hyperon in this process will only lead to further suppression, the additional contribution to the dark luminosity from $\Lambda$-bremsstrahlung can be neglected.

\section{REABSORPTION AND TRAPPING}

The emitted $X^{0}$ can get reabsorbed by the stellar medium if their mean-free path is shorter than the size of the PNS [53,54,57,64-66]. The main absorption mechanism is the inverse of production, $X^{0} n \rightarrow \Lambda$, and the absorption rate per unit volume $d \mathcal{N}_{\mathrm{ab}} / d \omega$ can be calculated similarly to the emission rate. Assuming time-reversal implies that the matrix elements of both processes are equal while thermal equilibrium implies that $\left(1+f_{X}\right) f_{\Lambda}\left(1-f_{n}\right)=$ $f_{X}\left(1-f_{\Lambda}\right) f_{n}$. Thus,

$$
\frac{d \mathcal{N}_{\mathrm{ab}}}{d \omega}=\frac{d \mathcal{N}_{\mathrm{em}}}{d \omega},
$$

which is just the detailed balance between emission and absorption [64-66]. From Eq. (6) it is straightforward to calculate the energy-dependent mean-free path $\lambda_{\omega}$ as

$$
\lambda_{\omega}^{-1}=\frac{1}{\frac{d n_{X}}{d \omega}} \frac{d \mathcal{N}_{\mathrm{ab}}}{d \omega}=\frac{m_{\Lambda}^{2} \Gamma}{\bar{\omega} \omega^{2}} \int_{E_{0}}^{\infty} d E\left(1-f_{\Lambda}\right) f_{n},
$$

where $n_{X}$ is the number density of $X^{0}$ in the medium. The flux of $X^{0}$ with energy $\omega$ that propagate outwards in the PNS from a point at radius $r$ will experience an exponential damping from absorption described by the optical depth,

$$
\tau(\omega, r)=\int_{r}^{\infty} \lambda_{\omega}\left(r^{\prime}\right)^{-1} d r^{\prime}
$$

where the mean-free path depends on the thermodynamical quantities at $r^{\prime}$. The total dark luminosity of the PNS can be then written as,

$$
L_{\mathrm{d}}=\int d^{3} \vec{r} \int_{0}^{\infty} d \omega \frac{d Q(r)}{d \omega} e^{-\tau(\omega, r)},
$$

where the energy-loss rate also depends on the radius.

This equation describes the attenuation of the flux by reabsorption but it does not account completely for the 
luminosity in the strong $X^{0}$-coupling limit, where the mean free path becomes much shorter than the radius of the PNS and the $X^{0}$ undergoes multiple absorptions and emissions before leaving the star. In this trapping regime, emission of $X^{0}$ is better described by black-body radiation from a surface where the optical depth is, averaged over $\omega$, equal to $2 / 3[64,65]$. In our case there is a maximum radius of emission, $R_{\mathrm{d}}$, at density and temperature $\left(T_{\mathrm{d}}\right)$ such that $\Lambda$ 's are not longer produced in the medium. This sets a minimal emission loss-rate in the trapping regime determined by

$$
L_{\mathrm{d}}^{\mathrm{t}}=\frac{\pi^{3}}{30} g_{s} R_{\mathrm{d}}^{2} T_{\mathrm{d}}^{4}
$$

where $g_{s}$ the spin-degeneracy factor of the $X^{0}$.

\section{SUPERNOVA SIMULATIONS AND EOS}

A robust computation of the dark luminosity with the equations above requires knowing the radial profiles of the relevant thermodynamical quantities at a given time of the $\mathrm{SN}$ explosion. We use recent simulations including muons that were developed specifically to constrain the axionmuon coupling using the neutrino data from SN 1987A [40]. Two EoS are employed for nuclear matter, SFHo [67] and LS220 [68], and the simulations are performed for different masses of the neutron star spanning the range allowed by observations [69]. These are labeled by SFHo18.8, SFHo-18.6 and SFHo-20.0, or by LS220-20.0, depending on the EoS and mass of the progenitor star (in solar masses) used. The simulations are spherically symmetric (one-dimensional) and explosions are, therefore, artificially triggered [40,46,70-72]. The data consist of radial profiles of different thermodynamical variables such as density, temperature and the particle abundancies at various post-bounce times [73].

Hyperons are not included as particle ingredients in the simulations. However, they can be added indirectly through the nuclear EoS because the SFHo and LS220 models have been extended with $\Lambda$ 's as explicit degrees of freedom; these EoS are called SFHoY [74] and LS220 $[75,76]$, respectively. In case of SFHo, the hyperonic and nonhyperonic EoS lead to almost identical predictions of the system's thermodynamical properties for all the conditions reached in the SN simulations [74] (they are also consistent with all known nuclear and astrophysical constraints [39]). Thus, one expects that their results (radial profiles) are not affected by the inclusion of hyperons. In case of LS220, on the other hand, differences between the two types of EoS start occurring at twice nuclear density [77], which are conditions reached at the core of the PNS. The LS220 $\Lambda$ EoS is also unable to produce neutron stars with $2 M_{\odot}$ masses, being in conflict with observations [78-80]. Therefore, in our analysis, we use the results from the SFHo simulations as our baseline and include LS220 only

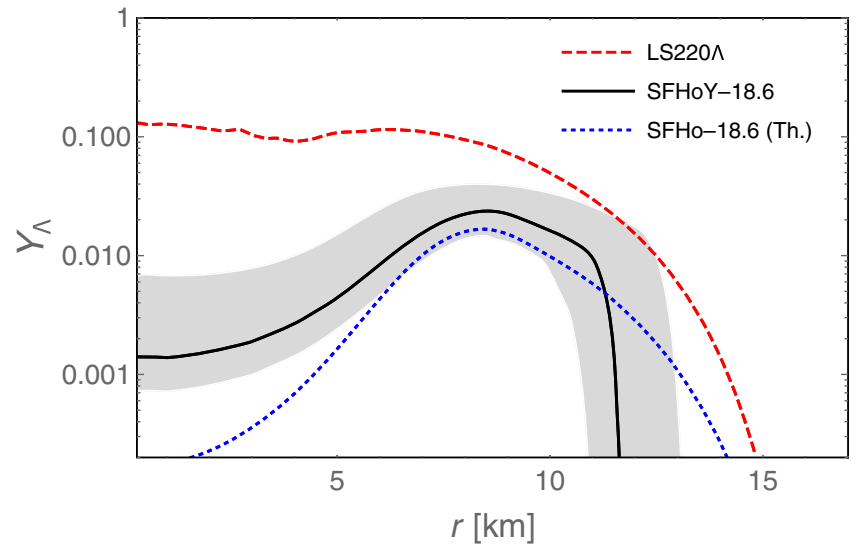

FIG. 1. Radial profiles of the $\Lambda$ abundancy $Y_{\Lambda}=n_{\Lambda} / n_{B}$, with $n_{B}$ the baryon number density, for the simulations of [40] at $\sim 1 \mathrm{~s}$ after bounce and using the appropriate extensions of the nuclear LS220 (red dashed) and SFHo EoS with hyperons [81]. The upper and lower limits of the gray band around the SFHoY-18.6 curve (solid black) correspond to the SFHo simulations with $20 M_{\odot}$ and $18.8 M_{\odot}$, respectively. We also include the results for SFHo-18.6 assuming a purely thermal distribution (blue dotted), see Eq. (5).

to test the robustness of the results with respect to the choice of EoS.

We use the radial profiles of density, temperature and proton fraction from the simulations as inputs to obtain the radial profiles of the other relevant thermodynamical quantities, which are derived from interpolation tables generated by the CompOSE database [81]. In Fig. 1 we show the profiles of the $\Lambda$ abundancy, $Y_{\Lambda}$, predicted by these EoS for the simulations of [40] at $\sim 1 \mathrm{~s}$ post-bounce. A large abundancy of $\Lambda$, of the order of $10 \%$ is obtained at the core of the PNS for LS220 $\Lambda$. In case of SFHoY, $\Lambda$ abundancies are always modest, of less than a few percent, with a maximum at $r \simeq 7-8 \mathrm{~km}$ where the PNS reaches the highest temperatures and $\Lambda$-production is dominated by thermal effects.

\section{MEDIUM EFFECTS}

SFHoY implements a relativistic microscopic model with baryon-baryon interactions mediated by meson fields that are described in a mean-field approximation. For a baryon $\mathfrak{B}$ with three-momentum $\vec{p}$, the medium corrections lead to effective masses, $m_{\mathfrak{B}}^{*}$, and energies, $E_{\mathfrak{B}}^{*}=$ $\sqrt{\vec{p}^{2}+m_{\mathfrak{B}}^{* 2}}+V_{\mathfrak{B}}$, where $V_{\mathfrak{B}}$ is the timelike component of the vector self-energy [82]. In the case of LS, the baryonbaryon interactions are modeled using nonrelativistic effective interactions. The in-medium masses are not modified while the energies receive a contribution similar to $V_{\mathfrak{B}}$ but adopting the form of a nonrelativistic potential $[68,75]$.

These medium modifications have to be taken into account in the distributions $f_{\mathfrak{B}}$ in order to obtain the right 
baryon abundancies [81], and in the calculation of the emission and absorption rates. These simplify considerably if we neglect $V_{n}-V_{\Lambda}$, which is $\sim 10 \mathrm{MeV}$ for all relevant conditions. In the Supplemental Material [83]we present the corresponding formulas for $d Q / d \omega$ and $\lambda_{\omega}^{-1}$.

\section{RESULTS}

Combining all the previous ingredients we compute the dark luminosity of SN 1987A as a function of $\Gamma\left(\Lambda \rightarrow n X^{0}\right)$. Comparing this to the bound in luminosity shown in Eq. (1) allows us to set an upper limit on the branching fraction of the decay $\Lambda \rightarrow n X^{0}$. In Table I we collect our results for the various SN simulations (evaluated at $1 \mathrm{~s}$ after bounce) and different approaches to calculate the rates. "Thermal" and "EoS-App." employ the approximate Eq. (4) in combination with either Eq. (5) or the corresponding hyperonic EoS for $n_{\Lambda}$, respectively. "EoS" is obtained from exactly solving Eq. (3) and including medium effects $\left(m_{\mathfrak{B}}^{*}\right.$ and $\left.V_{\mathfrak{B}}\right)$ in the calculation of the $f_{\mathfrak{B}}$. In "EoS*" we also include these effects in the calculation of the rates.

We find that the bounds are quite robust with respect to the approach used for the calculation of the luminosities. The largest difference we find is by a factor $\sim 2$ in some simulations. On the other hand, they are very sensitive to the mass of the neutron star, with differences that can be larger than by an order of magnitude. The simulations collapsing $20 M_{\odot}$ provide markedly stronger limits as they are the ones where the PNS reach the highest temperatures and densities [40]. Although LS220 $\Lambda$ predicts a larger number of $\Lambda$ 's it eventually leads to only slightly stronger limits compared to SFHoY-20.0.

In the last row of Table I we also show the minimal luminosities obtained in the trapping regime, see Eq. (10), which are all much larger than the upper limit in Eq. (1). This is due to the fact that the last surface of the PNS where $\Lambda$ 's can be produced in equilibrium corresponds to a very hot region. On the other hand, the $X^{0}$ can still be trapped in the PNS if one adds sufficiently strong interactions with nucleons (star cooling bounds actually prevent trapping

TABLE I. Upper limits on $\operatorname{BR}\left(\Lambda \rightarrow n X^{0}\right)$, in units of $10^{-10}$, for different $\mathrm{SN}$ simulations and approaches in the calculation of the dark emissivity (see main text). The value in boldface corresponds to our baseline result. In the last row we show the minimal emissivity achieved in the trapping regime (for $g_{s}=1$ ).

\begin{tabular}{lcccc}
\hline \hline & SFHo-18.6 & SFHo-18.8 & SFHo-20.0 & LS220-20.0 \\
\hline Thermal & 27 & 60 & 7 & 6 \\
EoS-App. & 20 & 46 & 6 & 2 \\
EoS & 36 & 92 & 10 & 4 \\
EoS $^{*}$ & 32 & $\mathbf{8 1}$ & 9 & 4 \\
$L_{\mathrm{d}}^{\mathrm{t}}\left[\mathrm{erg} \mathrm{s}^{-1}\right]$ & $1.1 \times 10^{55}$ & $6.5 \times 10^{54}$ & $1.7 \times 10^{55}$ & $1.7 \times 10^{54}$ \\
\hline \hline
\end{tabular}

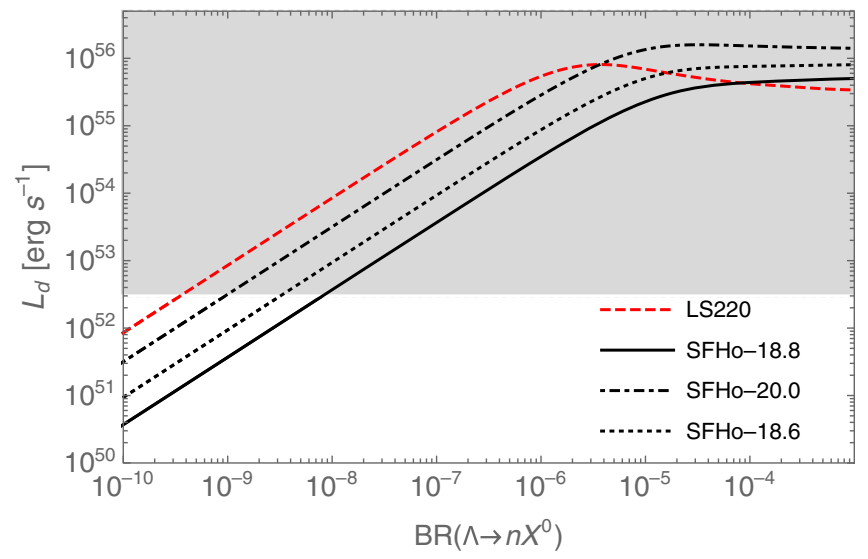

FIG. 2. Dark luminosity with EoS* for the various simulations at $\sim 1$ s post-bounce as a function of the branching-fraction of the decay $\Lambda \rightarrow n X^{0}$. Gray region is excluded by Eq. (1).

with electrons [30]). However, the large couplings needed for this are typically excluded by other constraints.

In Fig. 2 we show the dependence of the luminosity on the branching fraction for the different simulations and including medium corrections to the rates. The flattening of the curves at large coupling (or branching ratio) reflects the behavior in the trapping regime discussed previously. Given all the above, the SN 1987A bound is,

$$
\operatorname{BR}\left(\Lambda \rightarrow n X^{0}\right) \lesssim 8.0 \times 10^{-9},
$$

obtained by combining the most refined calculation (EoS*) with the simulation giving the weaker bound (SFHo-18.80) [40]. Note that this is a conservative limit because it stems from the simulation that produces the SN 1987As remnant on the low-mass edge of the allowed range and, therefore, have the coolest profile. If we were to use SFHo-20.0 (on the high-mass end of this range) we would get an order-ofmagnitude stronger bound, $\operatorname{BR}\left(\Lambda \rightarrow n X^{0}\right) \lesssim 9.0 \times 10^{-10}$. (See also ref. [84] for a more refined statistical approach). Finally, let us stress that our constraint is model independent in the sense that it applies to any ultralight dark particle inducing the $\Lambda$ decay and long-lived enough to leave the PNS.

\section{DARK PHOTONS}

In order to apply our result to the massless dark photon case we consider the dimension-five operator

$$
\mathcal{L}_{\gamma^{\prime}}=\frac{1}{\Lambda_{\mathrm{UV}}} \bar{\psi}_{i} \sigma^{\mu \nu}\left(\mathbb{C}^{i j}+i \mathbb{C}_{5}^{i j} \gamma_{5}\right) \psi_{j} F_{\mu \nu}^{\prime},
$$

where $F_{\mu \nu}^{\prime}$ is the field strength associated to the dark photon, $\psi_{i}$ are the SM fermions and $\mathbb{C}_{(5)}^{i j}$ are the couplings of the interaction, suppressed by the energy scale $\Lambda_{\mathrm{UV}}$, that depends on the underlying UV completion [3]. This operator allows for flavor off-diagonal couplings and 
would contribute to the dark width in Eq. (2) with $C_{\gamma^{\prime}}=8 g_{T}^{2} / \Lambda_{\mathrm{UV}}^{2}\left(\left|\mathbb{C}^{d s}\right|^{2}+\left|\mathbb{C}_{5}^{d s}\right|^{2}\right)$, where $g_{T}$ is the $\Lambda \rightarrow n$ tensor charge. We use the value $g_{T}=-0.73$ which is obtained by using SU(3)-flavor symmetry with the tensor charges of the nucleon calculated in the lattice $[85,86]$ (see Supplemental Material [83] for details).

Taking the upper limit on $\mathrm{BR}\left(\Lambda \rightarrow n X^{0}\right)$ by $\mathrm{SN}$ given in Eq. (11) we can set the lower limit,

$$
\Lambda_{\mathrm{UV}} \gtrsim 1.2 \times 10^{10} \mathrm{GeV}
$$

assuming order-one couplings. This can be compared to the limits on flavor-violating hyperon decays from laboratory experiments. Using the upper bounds on the invisible branching fractions given in Table II of [29] and the tensor form factors in the Supplemental Material [83], the decay $\Xi^{0} \rightarrow \Sigma^{0} \gamma^{\prime}$ sets the strongest limit, $\Lambda_{\mathrm{UV}} \gtrsim 4.3 \times 10^{7} \mathrm{GeV}$. This could be improved in future experiments like BESIII. Using the prospected sensitivity for $\operatorname{BR}\left(\Lambda \rightarrow n \gamma^{\prime}\right) \simeq$ $\operatorname{BR}(\Lambda \rightarrow n \nu \bar{\nu})$ [87], the bound could be pushed up to $1.9 \times 10^{9} \mathrm{GeV}$. Kaon decays can place a similar limit using $\operatorname{BR}\left(K^{+} \rightarrow \pi^{+} \pi^{0} X^{0}\right)$ [88]; applying the calculations derived in [23] we get $\Lambda_{\mathrm{UV}} \gtrsim 1.7 \times 10^{7} \mathrm{GeV}$. Probing this beyond the SN limit, for example at the NA62 experiment [20], would require reaching a sensitivity $\operatorname{BR}\left(K^{+} \rightarrow \pi^{+} \pi^{0} X^{0}\right) \lesssim$ $1.85 \times 10^{-10}$.

We can also compare with bounds on dark photon couplings to other matter fields, which have been collected in Ref. [3]. In Fig. 3 we show these limits together with the new SN bound on $d s$-couplings (note that we have updated the SN bounds on nucleons using Ref. [62]). We see that the SN analysis done in this work sets the strongest limit on dark photon couplings, along with star cooling constraints on lepton couplings. This bound cannot be

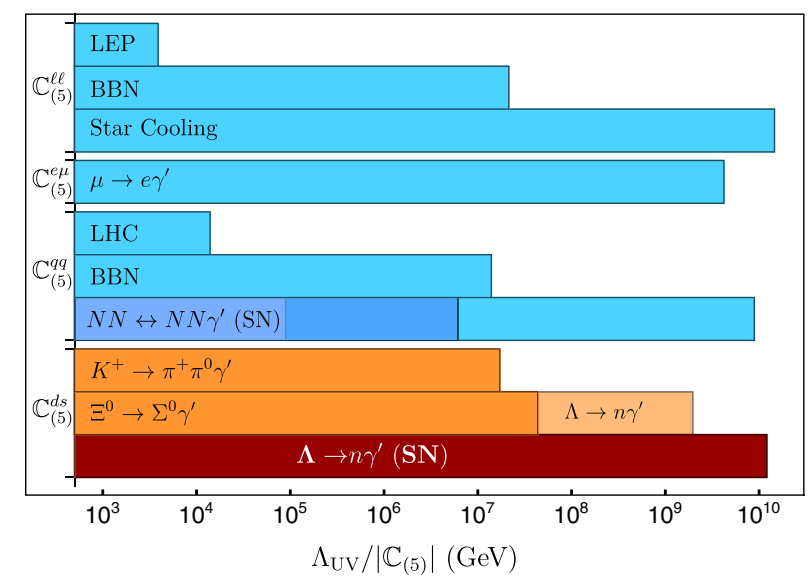

FIG. 3. Model independent excluded region of the couplings of the dipole operator Eq. (12) from various sources. In blue, limits to quark flavor diagonal and lepton couplings; in orange, limits to $d s$ coupling that can be directly compared to the one derived in this work, in dark red. In faint orange the prospected limit from BESIII. avoided by trapping the dark photon with large couplings to nucleons, since constraints from SN 1987A close the entire window, see Fig. 3. This results from combining the luminosity constraint (light blue) with the absence of a signal in the Kamiokande detector (dark blue).

\section{AXIONS}

The couplings of axions to SM fields are

$$
\mathcal{L}_{a}=\frac{\partial_{\mu} a}{2 f_{a}} \bar{\psi}_{i} \gamma^{\mu}\left(c_{i j}^{V}+c_{i j}^{A} \gamma_{5}\right) \psi_{j}
$$

where $a$ is the axion field and $f_{a}$ is its decay constant. The axion contribution to Eq. (2) is given by $C_{a}=$ $\left(f_{1}^{2}\left|c_{d s}^{V}\right|^{2}+g_{1}^{2}\left|c_{d s}^{A}\right|^{2}\right) /\left(2 f_{a}\right)^{2}$, where $f_{1}$ and $g_{1}$ are form factors that are discussed in the Supplemental Material [83]. Using the values shown there and the SN limit in Eq. (11) we obtain,

$F_{s d}^{V} \gtrsim 7.1 \times 10^{9} \mathrm{GeV}, \quad F_{s d}^{A} \gtrsim 5.2 \times 10^{9} \mathrm{GeV}$,

for pure vector and axial couplings $F_{s d}^{V, A} \equiv 2 f_{a} / c_{s d}^{V, A}$, respectively. A comprehensive discussion of other bounds in this model can be found in [29]. In particular, this constraint is also stronger than the SN bounds on the diagonal couplings to light quarks from nucleon-nucleon bremsstrahlung, and on the leptonic couplings to $\mu \mu$ [40] and $\mu e$ [30]. Finally, as we discuss in detail in the Supplemental Material [83], the bounds for the QCD axion are roughly applicable also to the ALP case unless its mass is very close to the $\Lambda-n$ mass difference. It is interesting to note that the $\mathrm{SN}$ bound on ALPs can become comparable to the stringent bounds from laboratory experiments looking for $K^{+} \rightarrow \pi^{+} X^{0}$ in the two-pion decay region, where the sensitivity is strongly reduced due to the SM background [89].

\section{CONCLUSIONS}

We have studied in detail a novel SN bound on dark flavored sectors stemming from the decay of $\Lambda$ hyperons in the proto-neutron star. We have used state-of-the-art simulations with the corresponding hyperonic EoS for our calculations to obtain the upper limit $\operatorname{BR}\left(\Lambda \rightarrow n X^{0}\right) \lesssim$ $8.0 \times 10^{-9}$. This leads to the strongest bounds that have been derived so far on the couplings of the massless dark photon to quarks. This analysis also sets strong constraints on flavor-violating axion models, and can be readily extended to other flavored dark sectors.

\section{ACKNOWLEDGMENTS}

We thank R. Bollig, H.-Th. Janka and M. Oertel for useful discussions and for providing us with internal details of their work. We also thank F.S. Kitaura for useful 
discussions. J.M.C. acknowledges support from the Spanish MINECO through the "Ramón y Cajal" Program No. RYC-2016-20672 and the Grant No. PGC2018-102016-A-I00. The work of J. T.C. is supported by the Ministerio de Ciencia e Innovación under FPI Contract No. PRE2019-089992 of the SEV-2015-0548 grant and Generalitat Valenciana by the SEJI/2018/033 project. The work of L.T. is supported by Contract No. FPA2016-81114-P financed by: Ministerio de Economía y Competitividad-Agencia Estatal de
Investigación-FEDER, UE and Contract No. PID2019110165GB-I00 financed by: Ministerio de Ciencia e Innovación-Agencia Estatal de Investigación-FEDER, UE, by PHAROS COST Action CA16214, and by the EU STRONG-2020 project under the program H2020INFRAIA-2018-1, Grant agreement No. 824093. This work is partially supported by project C3b of the DFGfunded Collaborative Research Center TRR257, "Particle Physics Phenomenology after the Higgs Discovery".
[1] J. Silk et al., in Particle Dark Matter: Observations, Models and Searches, edited by G. Bertone (Cambridge University Press, Cambridge, England, 2010).

[2] B. Holdom, Phys. Lett. 166B, 196 (1986).

[3] M. Fabbrichesi, E. Gabrielli, and G. Lanfranchi, arXiv: 2005.01515.

[4] R. D. Peccei and H. R. Quinn, Phys. Rev. Lett. 38, 1440 (1977).

[5] R. D. Peccei and H. R. Quinn, Phys. Rev. D 16, 1791 (1977).

[6] F. Wilczek, Phys. Rev. Lett. 40, 279 (1978).

[7] S. Weinberg, Phys. Rev. Lett. 40, 223 (1978).

[8] A. Arvanitaki, S. Dimopoulos, S. Dubovsky, N. Kaloper, and J. March-Russell, Phys. Rev. D 81, 123530 (2010).

[9] J. E. Kim and G. Carosi, Rev. Mod. Phys. 82, 557 (2010); 91, 049902(E) (2019).

[10] J. Jaeckel and A. Ringwald, Annu. Rev. Nucl. Part. Sci. 60, 405 (2010).

[11] L. Di Luzio, M. Giannotti, E. Nardi, and L. Visinelli, Phys. Rep. 870, 1 (2020).

[12] G. Lanfranchi, M. Pospelov, and P. Schuster, arXiv: 2011.02157.

[13] J. Alexander et al., arXiv:1608.08632.

[14] M. Battaglieri et al., in U.S. Cosmic Visions: New Ideas in Dark Matter (Maryland University, College Park, MD, 2017) [arXiv:1707.04591].

[15] J. Alimena et al., J. Phys. G 47, 090501 (2020).

[16] J. Beacham et al., J. Phys. G 47, 010501 (2020).

[17] B. A. Dobrescu, Phys. Rev. Lett. 94, 151802 (2005).

[18] J. F. Kamenik and C. Smith, J. High Energy Phys. 03 (2012) 090.

[19] E. Gabrielli, B. Mele, M. Raidal, and E. Venturini, Phys. Rev. D 94, 115013 (2016).

[20] M. Fabbrichesi, E. Gabrielli, and B. Mele, Phys. Rev. Lett. 119, 031801 (2017).

[21] M. Fabbrichesi and E. Gabrielli, Eur. Phys. J. C 80, 532 (2020).

[22] J.-Y. Su and J. Tandean, Phys. Rev. D 101, 035044 (2020).

[23] J.-Y. Su and J. Tandean, Eur. Phys. J. C 80, 824 (2020).

[24] F. Wilczek, Phys. Rev. Lett. 49, 1549 (1982).

[25] J. L. Feng, T. Moroi, H. Murayama, and E. Schnapka, Phys. Rev. D 57, 5875 (1998).
[26] L. Calibbi, F. Goertz, D. Redigolo, R. Ziegler, and J. Zupan, Phys. Rev. D 95, 095009 (2017).

[27] Y. Ema, K. Hamaguchi, T. Moroi, and K. Nakayama, J. High Energy Phys. 01 (2017) 096.

[28] F. Björkeroth, E. J. Chun, and S. F. King, J. High Energy Phys. 08 (2018) 117.

[29] J. M. Camalich, M. Pospelov, P. N. H. Vuong, R. Ziegler, and J. Zupan, Phys. Rev. D 102, 015023 (2020).

[30] L. Calibbi, D. Redigolo, R. Ziegler, and J. Zupan, arXiv:2006.04795.

[31] K. Sato and H. Sato, Prog. Theor. Phys. 54, 1564 (1975).

[32] M. Vysotsky, Y. Zeldovich, M. Khlopov, and V. Chechetkin, Pis'ma Zh. Eksp. Teor. Fiz. 27, 533 (1978).

[33] D. A. Dicus, E. W. Kolb, V. L. Teplitz, and R. V. Wagoner, Phys. Rev. D 18, 1829 (1978).

[34] D. A. Dicus, E. W. Kolb, V. L. Teplitz, and R. V. Wagoner, Phys. Rev. D 22, 839 (1980).

[35] G. Raffelt, Stars as Laboratories for Fundamental Physics: The Astrophysics of Neutrinos, Axions, and Other Weakly Interacting Particles (University Of Chicago Press, Chicago, 1996).

[36] P. Zyla et al. (Particle Data Group), Prog. Theor. Exp. Phys. 2020, 083C01 (2020).

[37] H.-T. Janka, K. Langanke, A. Marek, G. Martinez-Pinedo, and B. Mueller, Phys. Rep. 442, 38 (2007).

[38] R. Bollig, H. T. Janka, A. Lohs, G. Martinez-Pinedo, C. Horowitz, and T. Melson, Phys. Rev. Lett. 119, 242702 (2017).

[39] M. Oertel, M. Hempel, T. Klähn, and S. Typel, Rev. Mod. Phys. 89, 015007 (2017).

[40] R. Bollig, W. DeRocco, P. W. Graham, and H.-T. Janka, Phys. Rev. Lett. 125, 051104 (2020).

[41] D. Croon, G. Elor, R. K. Leane, and S. D. McDermott, J. High Energy Phys. 01 (2021) 107.

[42] P. Cigan et al., Astrophys. J. 886, 51 (2019).

[43] D. Page, M. V. Beznogov, I. Garibay, J. M. Lattimer, M. Prakash, and H.-T. Janka, Astrophys. J. 898, 125 (2020).

[44] A. Burrows and J. M. Lattimer, Astrophys. J. 307, 178 (1986).

[45] H. Bethe, Rev. Mod. Phys. 62, 801 (1990).

[46] H.-T. Janka, Annu. Rev. Nucl. Part. Sci. 62, 407 (2012). 
[47] N. Bar, K. Blum, and G. D'Amico, Phys. Rev. D 101, 123025 (2020).

[48] T. J. Loredo and D. Q. Lamb, Phys. Rev. D 65, 063002 (2002).

[49] F. Vissani, J. Phys. G 42, 013001 (2015).

[50] R. Bionta et al., Phys. Rev. Lett. 58, 1494 (1987).

[51] K. Hirata et al. (Kamiokande-II Collaboration), Phys. Rev. Lett. 58, 1490 (1987).

[52] E. Alekseev, L. Alekseeva, I. Krivosheina, and V. Volchenko, Phys. Lett. B 205, 209 (1988).

[53] G. Raffelt and D. Seckel, Phys. Rev. Lett. 60, 1793 (1988).

[54] M. S. Turner, Phys. Rev. Lett. 60, 1797 (1988).

[55] R. Mayle, J. R. Wilson, J. R. Ellis, K. A. Olive, D. N. Schramm, and G. Steigman, Phys. Lett. B 203, 188 (1988).

[56] A. Burrows, M. S. Turner, and R. Brinkmann, Phys. Rev. D 39, 1020 (1989).

[57] A. Burrows, M. Ressell, and M. S. Turner, Phys. Rev. D 42, 3297 (1990).

[58] C. Hanhart, D. R. Phillips, and S. Reddy, Phys. Lett. B 499, 9 (2001).

[59] J. B. Dent, F. Ferrer, and L. M. Krauss, arXiv:1201.2683.

[60] E. Rrapaj and S. Reddy, Phys. Rev. C 94, 045805 (2016).

[61] J. H. Chang, R. Essig, and S. D. McDermott, J. High Energy Phys. 09 (2018) 051.

[62] P. Carenza, T. Fischer, M. Giannotti, G. Guo, G. MartínezPinedo, and A. Mirizzi, J. Cosmol. Astropart. Phys. 10 (2019) 016; 05 (2020) E01.

[63] P. Carenza, B. Fore, M. Giannotti, A. Mirizzi, and S. Reddy, Phys. Rev. Lett. 126, 071102 (2021).

[64] Y. B. Zel'dovich and Y. P. Raizer, Physics of Shock Waves and High-Temperature Hydrodynamic Phenomena (Dover Publications, Inc., Mineola, 1967).

[65] S. Chandrasekhar, An Introduction to the Study of Stellar Structure (Dover Publications, Inc., 1967).

[66] R. Kippenhahn, A. Weigert, and A. Weiss, Stellar Structure and Evolution (Springer-Verlag, Berlin, 2012).

[67] A. W. Steiner, M. Hempel, and T. Fischer, Astrophys. J. 774, 17 (2013).

[68] J. M. Lattimer and F. Swesty, Nucl. Phys. A535, 331 (1991).

[69] "SFH" stands for Steiner, Hempel and Fischer while "o" refers to their optimal model [67]. "LS" stands for Lattimer and Swesty and the number refers to the nuclear incompressibility $K=220 \mathrm{MeV}$ that is used [68]. See Ref. [39] for a review.

[70] M. Rampp, Ph.D. thesis, 1997.

[71] M. Rampp and H. Janka, Astron. Astrophys. 396, 361 (2002).

[72] A. Mirizzi, I. Tamborra, H.-T. Janka, N. Saviano, K. Scholberg, R. Bollig, L. Hudepohl, and S. Chakraborty, Riv. Nuovo Cimento 39, 1 (2016).

[73] Garching Core-Collapse Supernova Archive: https:// wwwmpa.mpa-garching.mpg.de/ccsnarchive/.

[74] M. Fortin, M. Oertel, and C. Providência, Pub. Astron. Soc. Aust. 35, e044 (2018).

[75] M. Oertel, A. Fantina, and J. Novak, Phys. Rev. C 85, 055806 (2012).

[76] F. Gulminelli, A. Raduta, M. Oertel, and J. Margueron, Phys. Rev. C 87, 055809 (2013).

[77] M. Oertel, F. Gulminelli, C. Providência, and A. R. Raduta, Eur. Phys. J. A 52, 50 (2016).

[78] P. Demorest, T. Pennucci, S. Ransom, M. Roberts, and J. Hessels, Nature (London) 467, 1081 (2010).

[79] J. Antoniadis et al., Science 340, 1233232 (2013).

[80] H. Cromartie et al. (NANOGrav Collaboration), Nat. Astron. 4, 72 (2020).

[81] CompStar Online Supernovæ-Equations of State: https:// compose.obspm.fr/.

[82] J. Walecka, Ann. Phys. (N.Y.) 83, 491 (1974).

[83] See Supplemental Material at http://link.aps.org/ supplemental/10.1103/PhysRevD.103.L121301 for details regarding the form factors, the calculation including medium and axion mass effects.

[84] C. Hanhart, J. A. Pons, D. R. Phillips, and S. Reddy, Phys. Lett. B 509, 1 (2001).

[85] R. Gupta, B. Yoon, T. Bhattacharya, V. Cirigliano, Y.-C. Jang, and H.-W. Lin, Phys. Rev. D 98, 091501 (2018).

[86] H. Georgi, Lie Algebras in Particle Physics. From Isospin to Unified Theories, Vol. 54 (CRC Press, Howard Georgi, 1982).

[87] H.-B. Li, Front. Phys. 12, 121301 (2017); 14, 64001(E) (2019).

[88] S. Adler et al. (E787 Collaboration), Phys. Rev. D 63, 032004 (2001).

[89] E. Cortina Gil et al. (NA62 Collaboration), J. High Energy Phys. 02 (2021) 201. 